Check for updates

Cite this: RSC Adv., 2018, 8, 36219

Received 6th September 2018

Accepted 7th October 2018

DOI: 10.1039/c8ra07429a

rsc.li/rsc-advances

\section{Electrochemiluminescence of gold nanoparticles and gold nanoparticle-labelled antibodies as co- reactants}

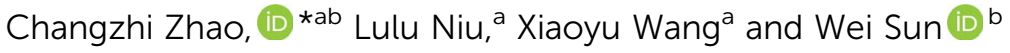 \\ Gold nanoparticles (AuNPs) and AuNP-labelled antibodies can participate in the electrochemiluminescence \\ $(E C L)$ reaction of tris $\left(2,2^{\prime}\right.$-bipyridyl) ruthenium(II) $\left(\mathrm{Ru}(\mathrm{bpy})_{3}{ }^{2+}\right)$ as a co-reactant. The electrochemical and \\ spectral characteristics of this new ECL system were confirmed by experiments, and its reaction \\ mechanism was speculated to be different from that of typical ECL. The experimental results showed \\ that the new ECL system exhibited higher ECL efficiency, and AuNP-labelled substances can be \\ sensitively measured with the help of AuNPs. Based on the dual role of AuNPs as a co-reactant and \\ marker, the AuNP-Ru(bpy) ${ }_{3}^{2+} \mathrm{ECL}$ system will be a useful tool in biochemical analysis.
}

\section{Introduction}

Electrochemiluminescence (ECL) is the process of releasing energy in the form of light radiation when unstable excited-state particles generated by some ECL reagents through the electrode reaction to complete a higher energy electron transfer return to the ground state. As the most common ECL reagent, ruthenium complex (such as $\mathrm{Ru}(\mathrm{bpy})_{3}{ }^{2+}$, where bpy $=2,2^{\prime}$-bipyridine) has been widely used in the fields of drug analysis, immunoassays and nucleic acid analysis because of its easy preparation, low costs and developed technology. ${ }^{1-3}$ The ECL reaction of $\mathrm{Ru}(\mathrm{bpy})_{3}{ }^{2+}$ requires a co-reactant in practical applications. The usual co-reaction reagents are reducing agents such as tripropylamine (TPrA), amino acids, ascorbic acid and quantum dots. $^{4-6}$ These reagents act as electron donors, allowing $\mathrm{Ru}(\mathrm{bpy})_{3}{ }^{3+}$ generated on the electrode to be reduced to produce excited state $\mathrm{Ru}(\mathrm{bpy})_{3}{ }^{2+} *$, but they cannot be used as a label at the same time.

Nanomaterials are substances having sizes between 1 and $100 \mathrm{~nm}$ and have at least one dimension in the threedimensional space. They have unique chemical and physical properties such as surface effects, micro-size effects, quantum effects and quantum tunneling effects; thus, they demonstrate superior properties that conventional materials do not have. ${ }^{7-9}$

Gold nanoparticles (AuNPs) are the most stable precious metal nanoparticles. Besides general nanomaterial properties, they also have high electron density, dielectric properties,

\footnotetext{
${ }^{a}$ Key Laboratory of Sensor Analysis of Tumor Marker, The Ministry of Education, College of Chemistry \& Molecular Engineering, Qingdao University of Science \& Technology, Qingdao 266042, China. E-mail: czzhao@qust.edu.cn

${ }^{b}$ Key Laboratory of Functional Materials and Photoelectronchemistry of Haikou, College of Chemistry and Chemical Engineering, Hainan Normal University, Haikou 571158, China. E-mail: swyy26@hotmail.com
}

catalytic properties and very good adsorption characteristics. $^{\mathbf{1 0 , 1 1}}$ In particular, AuNPs can combine with a variety of biological macromolecules without affecting their biological activity and are often used as carriers or markers in biochemical analysis. ${ }^{12-14}$ AuNP labelling technologies are one of the most advanced marking techniques. They have become a very useful tool in immunoassay and nucleic acid analysis and have been widely applied in food safety, environmental protection and clinical laboratories. ${ }^{15-17}$ However, methods to detect AuNPs or AuNP-labelled substances are limited to colorimetric, photometric, electron microscopy, and photomicrography with the help of visual methods. This results in difficulties in the accurate determination of AuNPs or AuNP-labelled substances. In addition, AuNPs have been applied in the ECL system as an ECL reagent or as a sensitizing material., ${ }^{\mathbf{4 1 8 , 1 9}}$ Although these reports indicate that AuNPs have good development prospects in ECL analysis, their role is constrained because of non-usage as a coreactant in ECL reactions. Here, we demonstrate that AuNPs can participate in ECL reaction as a co-reactant and have a good response in ECL reactions with $\mathrm{Ru}(\mathrm{bpy})_{3}{ }^{2+}$. Based on this discovery, AuNP-labelled antibodies can be determined, and the amount of immune complex can also be monitored, thereby gaining a very useful tool for biochemical analysis.

\section{Experimental}

\subsection{Materials and reagents}

Tris(2,2'-bipyridyl)ruthenium(II) chloride hexahydrate $\left(\mathrm{Ru}(\mathrm{bpy})_{3}{ }^{2+}\right)$ was purchased from Aldrich (Milwaukee, WI, USA). Its stock solution $\left(1.0 \times 10^{-3} \mathrm{~mol} \mathrm{~L}^{-1}\right)$ was prepared with $0.1 \mathrm{~mol} \mathrm{~L}^{-1}$ phosphate buffer (PBS) of $\mathrm{pH} 7.4$ and stored in a light-protected bottle. Working solutions of $\mathrm{Ru}(\mathrm{bpy})_{3}{ }^{2+}$ were freshly prepared from stock solutions with PBS containing $0.05 \mathrm{~mol} \mathrm{~L}^{-1} \mathrm{KNO}_{3}$. Standard gold nanoparticles and AuNP- 
labelled anti-IgG were obtained from cytodiagnostics (Cytodiagnostics lnc., Canada); their low concentration solutions were freshly prepared by diluting the standard solutions of nanoparticles with PBS before the experiment. All other chemicals were of analytical grade or higher quality. All solutions were prepared using ultrapure water (>18 M $\Omega$ ).

\subsection{Apparatus and measurements}

ECL measurements were performed with an MPI-B multifunctional ECL analyzer (Xi'an Remex Analytical Instrument Ltd. Co., China). The standard three electrode arrangements consisted of a CHI 104 glassy carbon disk electrode (GCE, $3.0 \mathrm{~mm}$ diameter), a CHI $111 \mathrm{Ag} / \mathrm{AgCl}$ reference electrode, and a CHI $115 \mathrm{Pt}$ counter electrode. The emission window of the ECL analyser was placed in front of a photomultiplier tube biased at $-800 \mathrm{~V}$. After the sample and electrodes were put in the electrolytic cell for $1 \mathrm{~min}$, the ECL response was obtained by scanning the potential across a suitable range. The ECL spectrum was obtained from potential scans of $\mathrm{Ru}(\mathrm{bpy})_{3}{ }^{2+}$ solutions containing AuNPs coupled with a CHI 660B electrochemical workstation (Shanghai Chenhua Instrument, Co., Ltd., China) and an F-2700 fluorescence spectrophotometer (HITACHI, Japan). The experiments were performed at room temperature. All measurements were repeated at least five times and means of the measurements were presented with the relative standard deviation (RSD).

\section{Results and discussion}

Gold is one of the most stable metals and has high resistance to oxidation and corrosion. Although the reduction potential of $\mathrm{Ru}(\mathrm{bpy})_{3}{ }^{3+}$ is as high as $1.20 \mathrm{~V},{ }^{20}$ it is still difficult to oxidize gold. Unlike the observations for bulk metals, the electronic structure of nanocrystals critically depends on their size. ${ }^{21}$ AuNPs, particularly small AuNPs, have more clear quantum effects and quantum tunnelling effects, which may result in a change of their electrochemical properties. ${ }^{22,23}$ An ECL spectrum (Fig. 1) was obtained from the potential scans of $\mathrm{Ru}(\mathrm{bpy})_{3}{ }^{2+}$ solutions containing $10 \mathrm{~nm}$ AuNPs by a luminometer coupled with an electrochemical workstation. As shown in Fig. 1, only one anode peak was observed at $1.13 \mathrm{~V}$, which was assigned to the oxidation of $\mathrm{Ru}(\mathrm{bpy})_{3}{ }^{3+}$. The maximum wavelength of ECL radiation was at $620 \mathrm{~nm}$ and its potential was

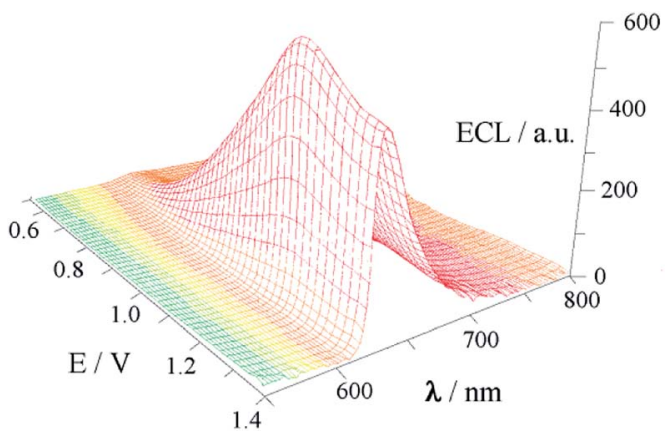

Fig. $1 \mathrm{ECL}$ spectrum of $10 \mathrm{~nm}$ AuNPs in $3.0 \times 10^{-4} \mu \mathrm{mol} \mathrm{mL} \mathrm{m}^{-1}$ $\mathrm{Ru}(\mathrm{bpy})_{3}{ }^{2+}$ solution. approximately $1.20 \mathrm{~V}$, which is consistent with the typical ECL characteristics of $\mathrm{Ru}(\mathrm{bpy})_{3}{ }^{2+} \cdot{ }^{3}$ These results indicate that AuNPs can participate in the ECL reaction of $\mathrm{Ru}(\mathrm{bpy})_{3}{ }^{2+}$ as a coreactant.

The typical ECL reaction between $\mathrm{Ru}(\mathrm{bpy})_{3}{ }^{2+}$ and its coreactants has been discussed extensively. ${ }^{4}$ The co-reactant of $\mathrm{Ru}(\mathrm{bpy})_{3}{ }^{2+}$ is usually a reducing agent in the ECL reaction, and it causes the reduction of $\mathrm{Ru}(\mathrm{bpy})_{3}{ }^{3+}$ to produce an excited state of $\mathrm{Ru}(\mathrm{bpy})_{3}{ }^{2+}$. TPrA, which is a commonly used co-reactant, releases electrons to undergo oxidation and becomes oxidized to $\mathrm{TPrA}^{+}$on the surface of the electrode, whereas $\mathrm{Ru}(\mathrm{bpy})_{3}{ }^{2+}$ is oxidized to $\mathrm{Ru}(\mathrm{bpy})_{3}{ }^{3+}$. Then, $\mathrm{TPrA}^{+}$spontaneously removes one proton to form the cation-excited state $\operatorname{TPrA}^{+}{ }^{\bullet}$, and the latter rapidly transfers electrons to $\mathrm{Ru}(\mathrm{bpy})_{3}{ }^{3+}$ to generate the excited state $\mathrm{Ru}(\mathrm{bpy})_{3}{ }^{2+*}$. Finally, unstable $\mathrm{Ru}(\mathrm{bpy})_{3}{ }^{2+*}$ releases light with a wavelength of $620 \mathrm{~nm}$. Due to gold being a solid material, its ECL reaction with $\mathrm{Ru}(\mathrm{bpy})_{3}{ }^{2+}$ is unlikely to be the same as TPrA regardless of its morphology. Assuredly, it is impossible for bulk $\mathrm{Au}$ to reduce $\mathrm{Ru}(\mathrm{bpy})_{3}{ }^{3+}$ because the potential of $\mathrm{AuCl}_{4}{ }^{-} / \mathrm{Au}_{\text {metal }}$ is as high as $1.0 \mathrm{~V} v$. $\mathrm{NHE}^{24}$ No reports on ECL enhancement of $\mathrm{Ru}(\mathrm{bpy})_{3}{ }^{2+}$ on bare gold electrodes in the absence of any co-reactants were found. Fortunately, the smallsize effects of AuNPs may give them different redox properties. An early report pointed out that gold-nanorod-modified electrodes exhibit an anodic peak at $0.81 \mathrm{~V} v s$. SCE, and this was due to the oxidation of the gold nanorod. ${ }^{25}$ Assuredly, a lower potential favours AuNP transfer of electrons to $\mathrm{Ru}(\mathrm{bpy})_{3}{ }^{3+}$. However, the change in the redox potential of AuNPs could be just a surface phenomenon, and a deeper reason may be obtained from its structure. The surface of AuNPs is an electric double layer structure, and the electric double layer capacitor acts as a nanometer-scale electrode. When the particle size of AuNPs is reduced to a certain extent, the electrostatic attraction energy becomes greater than the energy of thermal motion. Currently, electron transfer occurs between the counter ion (anti-charge material) that protects nano-gold and the electrode, resulting in charge and discharge of the electric double layer on the surface of AuNPs. ${ }^{23}$ The ECL reaction between AuNPs and $\mathrm{Ru}(\mathrm{bpy})_{3}{ }^{2+}$ is more consistent with this situation. Based on the above discussion, a possible mechanism of ECL reaction between AuNPs and $\mathrm{Ru}(\mathrm{bpy})_{3}{ }^{2+}$ is shown in Fig. 2 .

As shown in Fig. 2, after AuNPs were added to the electrolyte containing $\mathrm{Ru}(\mathrm{bpy})_{3}{ }^{2+}$, AuNPs with negative charges on their surface were immediately surrounded by $\mathrm{Ru}(\mathrm{bpy})_{3}{ }^{2+}$ due to electrostatic interactions to form an electric double layer. When

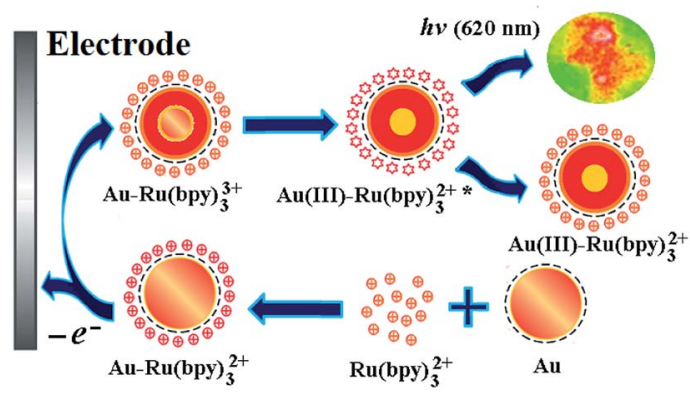

Fig. 2 ECL mechanism of reaction between AuNPs and Ru(bpy) ${ }_{3}^{2+}$. 
a suitable potential was applied to the electrode, the double layer particles with $\mathrm{Ru}(\mathrm{bpy})_{3}{ }^{2+}\left(\mathrm{Au}-\mathrm{Ru}(\mathrm{bpy})_{3}{ }^{2+}\right)$ diffused to the electrode surface and were oxidized to $\mathrm{Au}-\mathrm{Ru}(\mathrm{bpy})_{3}{ }^{3+}$. Then, $\mathrm{Ru}(\mathrm{bpy})_{3}{ }^{3+}$ captured electrons from the interior of AuNP double layer to generate an excited state. Finally, unstable $\mathrm{Au}(\mathrm{III})-$ $\mathrm{Ru}(\mathrm{bpy})_{3}{ }^{2+*}$ emitted light at $600 \mathrm{~nm}$. Using TPrA and AuNPs as co-reactants, the ECL intensities of their reaction with $\mathrm{Ru}(\mathrm{bpy})_{3}{ }^{2+}$ were determined under the same experimental conditions (Fig. 3). Compared to the result for the TPrA$\mathrm{Ru}(\mathrm{bpy})_{3}{ }^{2+}$ system, the ECL efficiency of $10 \mathrm{~nm}$ AuNPs was estimated to be 100000 times higher. Indeed, the AuNP solution is not a true solution, but even if AuNPs of $10 \mathrm{~nm}$ size contain 23800 gold atoms, ${ }^{26}$ the ECL efficiency caused by AuNPs is still 4 times that of TPrA. Further experimental results show that the ECL efficiency increases as the AuNP size decreases, which is consistent with the small-size effects of the nanoparticles. Although $5 \mathrm{~nm}$ AuNPs produce stronger ECL, practically, the most used AuNPs have sizes from 10 to $15 \mathrm{~nm}$. On the other hand, because of the particularity of AuNPs, other precious metal nanoparticles have not attracted our attention although we have found that silver nanoparticles react with $\mathrm{Ru}(\mathrm{bpy})_{3}{ }^{2+}$ to produce ECL.

The ECL system of AuNPs-Ru(bpy $)_{3}{ }^{2+}$ is beneficial for accurate determination of AuNP concentration. However, a more fascinating observation is that AuNPs can act as a marker and as a co-reactant at the same time in the ECL reaction. ECL responses of AuNPs and AuNP-labelled antibodies are shown in Fig. 4 in the presence of an equal amount of $\mathrm{Ru}(\mathrm{bpy})_{3}{ }^{2+}$. From Fig. 4, we observe that regardless of AuNPs or AuNP-labelled antibodies acting as co-reactants, the luminescence was almost the same and they both exhibited a clear response due to their same reaction mechanisms. The ECL intensity of $10 \mathrm{~nm}$ AuNPs was greater than that of $15 \mathrm{~nm}$ AuNPs (Fig. 4a) because of the small-size effects. As also seen from Fig. $4 \mathrm{~b}$, the AuNP$\mathrm{Ru}(\mathrm{bpy})_{3}{ }^{2+}$ system has a more sensitive response to the labeled antibody than AuNPs themselves, which is ascribed to the connection of each AuNP to several proteins.

AuNPs of 10-15 nm sizes have been widely applied in biological analysis as carriers or markers, and the antibodies labelled with them are mostly used in immunoassays. Using two antibodies labelled with 10- and $15 \mathrm{~nm}$ AuNPs as co-reactants,

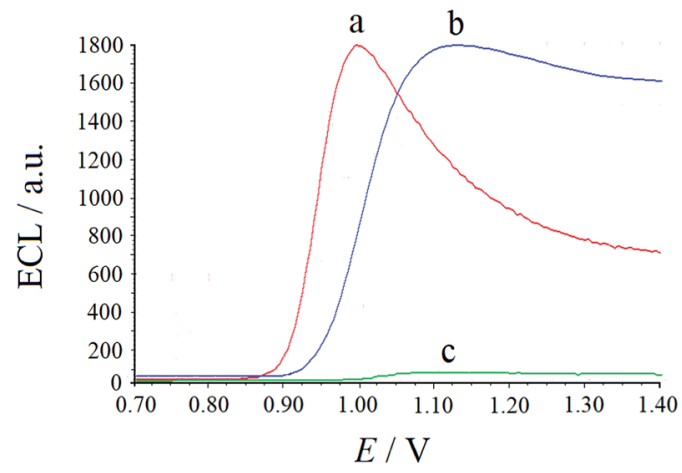

Fig. 3 ECL responses of $2.0 \times 10^{-4} \mathrm{mmol} \mathrm{mL}^{-1} \mathrm{Ru}(\mathrm{bpy})_{3}{ }^{2+}$ solution in the presence of $4.0 \times 10^{-4} \mu \mathrm{mol} \mathrm{mL}{ }^{-1} \operatorname{TPrA}(a), 6.8 \times 10^{-9} \mu \mathrm{mol} \mathrm{mL}^{-1}$ AuNPs (b) and absence (c).

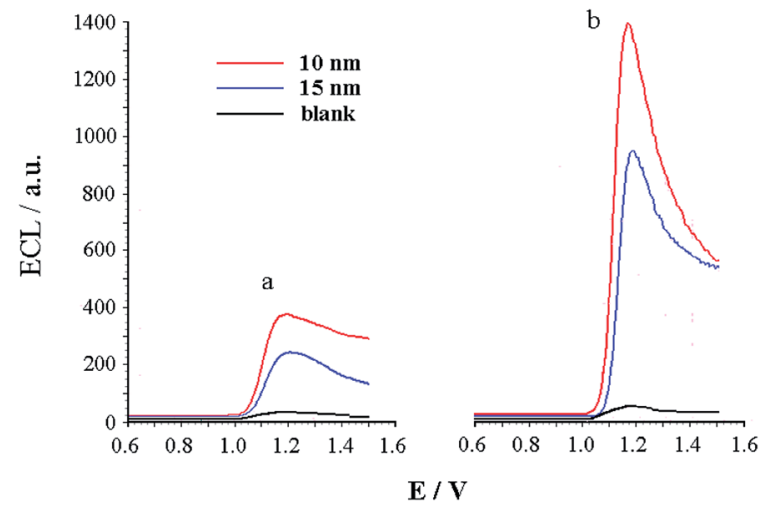

Fig. 4 ECL responses of $50 \mathrm{ng} \mathrm{mL}^{-1} 10$ and $15 \mathrm{~nm}$ AuNPs (a) and AuNPlabeled antibodies (b) in $2.0 \times 10^{-4} \mu \mathrm{mol} \mathrm{mL}{ }^{-1} \mathrm{Ru}(\mathrm{bpy})_{3}{ }^{2+}$ solution.

the performances for ECL reaction with $\mathrm{Ru}(\mathrm{bpy})_{3}{ }^{2+}$ are displayed in Fig. 5. As can be observed from Fig. 5, the antibodies exhibit a sensitive response with the help of the AuNP label. After observing two groups of curves a and $\mathrm{c}$ in the figure, we infer that ECL intensities increase as the concentration of the antibodies increases from 1.0 to $80 \mathrm{ng} \mathrm{mL}^{-1}$. The ECL signals show a linear response to the amount of labelled antibodies in the range of $5.0-80 \mathrm{ng} \mathrm{mL} \mathrm{m}^{-1}$ (Fig. $5 \mathrm{~b}$ and d), and the detection limit is less than $0.50 \mathrm{ng} \mathrm{mL}^{-1}$. It should be noted that the working electrode is not modified in our experiments, and the ECL reaction of the AuNP-labelled antibody with $\mathrm{Ru}(\mathrm{bpy})_{3}{ }^{2+}$ is carried out completely in solution. In an actual immunoassay, the labelled antibodies will be attached to the electrode surface, which may give the method a higher sensitivity.
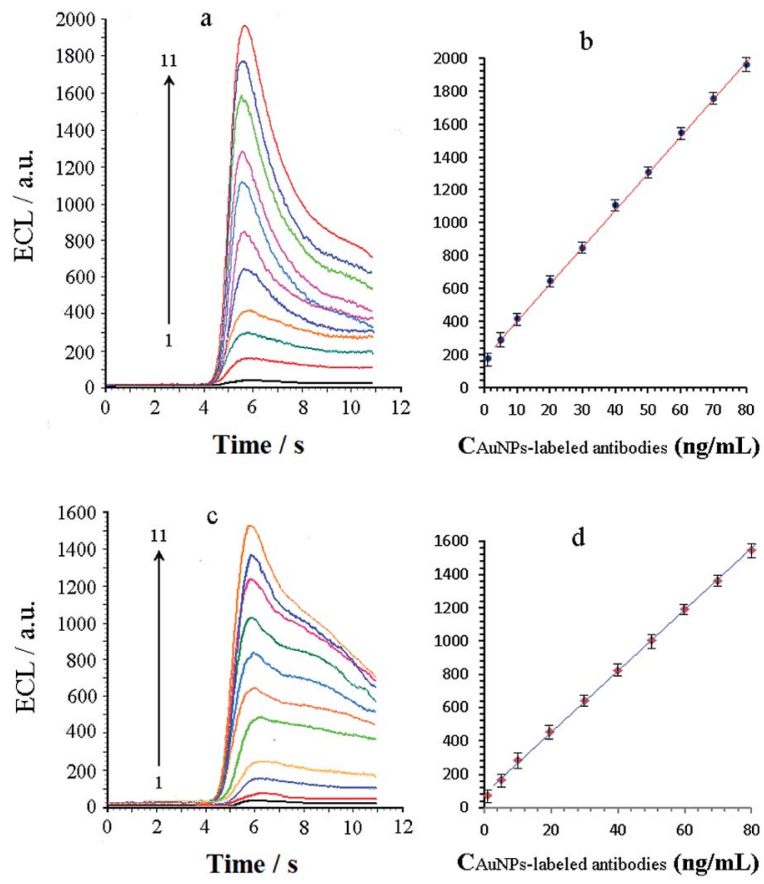

Fig. 5 ECL responses and calibration curve of various concentrations of antibodies labeled with 10 ( $a$ and b) or $15 \mathrm{~nm}$ (c and d) AuNPs in 2.0 $\times 10^{-4} \mu \mathrm{mol} \mathrm{mL}{ }^{-1} \mathrm{Ru}(\mathrm{bpy})_{3}{ }^{2+}$ solution, respectively. Concentration range (from bottom to top): 0, 1.00, 5.00, 10.0, 20.0, 30.0, 40.0, 50.0, 60.0, 70.0 and $80.0 \mathrm{ng} \mathrm{mL}^{-1}$. 


\section{Conclusions}

In summary, we have reported the ECL reaction of AuNPs and AuNP-labelled antibodies as a co-reactant. With the help of AuNPs, many AuNP-labeled substances can exhibit sensitive ECL responses. Thereby, the labelled substance or the substrate reacting with it can be monitored. Due to the combination of ECL and AuNP-labelling technology, the AuNP-Ru(bpy) ${ }_{3}{ }^{2+}$ system is expected to become the basis of some biochemical analyses.

\section{Conflicts of interest}

There are no conflicts to declare.

\section{Acknowledgements}

We acknowledge the financial support from the National Natural Science Foundation of Hainan Province (2017CXTD007), the Key Science Technology Program of Haikou City (2017042), and the Open-end Fund of Key Laboratory of Sensor Analysis of Tumor Marker, Ministry of Education (OESACLS201901).

\section{References}

1 G. Valenti, E. Rampazzo, S. Kesarkar, D. Genovese, A. Fiorani, A. Zanut, F. Palomba, M. Marcaccio, F. Paolucci and L. Prodi, Coord. Chem. Rev., 2018, 367, 65-81.

2 L. Li, Y. Chen and J.-J. Zhu, Anal. Chem., 2017, 89, 358-371. 3 M. M. Richter, Chem. Rev., 2004, 104, 3003-3036.

4 Y.-P. Dong, Y. Zhou, J. Wang and J.-J. Zhu, Anal. Chem., 2016, 88, 5469-5475.

5 P.-P. Dai, T. Yu, H.-W. Shi, J.-J. Xu and H.-Y. Chen, Anal. Chem., 2015, 87, 12372-12379.

6 Y. Yuan, S. Han, L. Hu, S. Parveen and G. Xu, Electrochim. Acta, 2012, 82, 484-492.

7 W.-W. Zhao, J. Wang, Y.-C. Zhu, J.-J. Xu and H.-Y. Chen, Anal. Chem., 2015, 87, 9520-9531.

8 C. Burda, X. Chen, R. Narayanan and M. A. El-Sayed, Chem. Rev., 2005, 105, 1025-1102.
9 C. M. Nieamyer, Science, 2002, 297, 62-63.

10 N. Elahi, M. Kamali and M. H. Baghersad, Talanta, 2018, 184, 537-556.

11 N. Siraj, B. El-Zahab, S. Hamdan, T. E. Karam, L. H. Haber, M. Li, S. O. Fakayode, S. Das, B. Valle, R. M. Strongin, G. Patonay, H. O. Sintim, G. A. Baker, A. Powe, M. Lowry, J. O. Karolin, C. D. Geddes and I. M. Warner, Anal. Chem., 2016, 88, 170-202.

12 Z. Farka, T. Jurík, D. Kovar, L. Trnkova and P. Skladal, Chem. Rev., 2017, 117, 9973-10042.

13 H. Xia, L. Li, Z. Yin, X. Hou and J.-J. Zhu, ACS Appl. Mater. Interfaces, 2015, 7, 696-703.

14 H. Liu, X. Wu, X. Zhang, C. Burda and J.-J. Zhu, J. Phys. Chem. $C, 2012,116,2548-2554$.

15 M. S. Draz and H. Shafiee, Theranostics, 2018, 8, 1985-2017. 16 E. A. Elbassal, C. Morris, T. W. Kent, R. Lantz, B. Ojha, E. P. Wojcikiewicz and D. J. Du, J. Phys. Chem. C, 2017, 121, 20007-20015.

17 T. Muthukumarasamyvel, G. Rajendran, D. S. Panneer, J. Kasthuri, K. Kathiravan and N. Rajendiran, ACS Omega, 2017, 2, 3527-3538.

18 Y.-M. Fang, J. Song, J. Li, Y.-W. Wang, H.-H. Yang, J.-J. Sun and G.-N. Chen, Chem. Commun., 2011, 47, 2369-2371.

19 L. Li, H. Liu, Y. Shen, J. Zhang and J.-J. Zhu, Anal. Chem., 2011, 83, 661-665.

20 C. R. Bock, J. A. Connor, A. R. Gutierrez, T. J. Meyer, D. G. Whitten, B. P. Sullivan and J. K. Nagle, J. Am. Chem. Soc., 1979, 101, 4815-4824.

21 C. N. R. Rao, G. U. Kulkarni, P. J. Thomas and P. P. Edwards, Chem.-Eur. J., 2002, 8, 28-35.

22 E. Gachard, H. Remita, J. Khatouri, B. Keita, L. Nadjo and J. Belloni, New J. Chem., 1998, 22, 1257-1265.

23 W. P. Halperin, Rev. Mod. Phys., 1986, 58, 533-606.

24 Lange's Handbook of Chemistry, ed. J. A. Dean, McGraw-Hill, New York, 15th edn, 1999, Section VII.

25 Y.-P. Dong, H. Cui and C.-M. Wang, J. Phys. Chem. B, 2006, 110, 18408-18414.

26 M. C. Scott, C.-C. Chen, M. Mecklenburg, C. Zhu, R. Xu, P. Ercius, U. Dahmen, B. C. Regan and J. Miao, Nature, 2012, 483, 444-491. 